OPEN ACCESS

Edited by:

Vadim V. Sumbayev,

University of Kent, United Kingdom

Reviewed by:

Luca Vanella,

Università degli Studi di Catania, Italy

Michael Danilenko,

Ben-Gurion University of the Negev,

Israel

*Correspondence:

Eui Kyun Park

epark@knu.ac.kr

${ }^{\dagger}$ These authors have contributed equally to this work

Specialty section: This article was submitted to Experimental Pharmacology

and Drug Discovery,

a section of the journal

Frontiers in Pharmacology

Received: 17 December 2018 Accepted: 25 March 2019

Published: 10 April 2019

Citation:

Ihn HJ, Lee T, Lee D, Bae J-S, Kim S-H, Jang IH, Bae YC, Shin H-I and Park EK (2019) Inhibitory Effect of KP-A038 on Osteoclastogenesis and Inflammatory Bone Loss is Associated With Downregulation of Blimp1. Front. Pharmacol. 10:367.

doi: 10.3389/fphar.2019.00367

\section{Inhibitory Effect of KP-A038 on Osteoclastogenesis and Inflammatory Bone Loss Is Associated With Downregulation
of Blimp1}

Hye Jung Ihn't, Taeho Lee ${ }^{2 t}$, Doohyun Lee ${ }^{2}$, Jong-Sup Bae', Sang-Hyun Kim ${ }^{3}$, II Ho Jang ${ }^{4}$, Yong Chul Bae ${ }^{5}$, Hong-In Shin ${ }^{6}$ and Eui Kyun Park ${ }^{6 *}$

1 Institute for Hard Tissue and Bio-tooth Regeneration, Kyungpook National University, Daegu, South Korea, ${ }^{2}$ College of Pharmacy, Research Institute of Pharmaceutical Sciences, Kyungpook National University, Daegu, South Korea, ${ }^{3}$ Department of Pharmacology, School of Medicine, Kyungpook National University, Daegu, South Korea, ${ }^{4}$ Department of Oral Biochemistry and Molecular Biology, School of Dentistry, Pusan National University, Yangsan, South Korea, ${ }^{5}$ Department of Oral Anatomy and Neurobiology, School of Dentistry, Kyungpook National University, Daegu, South Korea, ${ }^{6}$ Department of Oral Pathology and Regenerative Medicine, School of Dentistry, Institute for Hard Tissue and Bio-tooth Regeneration, Kyungpook National University, Daegu, South Korea

Excessive osteoclastic activity results in pathological bone resorptive diseases, such as osteoporosis, periodontitis, and rheumatoid arthritis. As imidazole-containing compounds possess extensive therapeutic potential for the management of diverse diseases, we synthesized a series of imidazole derivatives and investigated their effects on osteoclast differentiation and function. In the present study, we found that a novel imidazole derivative, KP-A038, suppressed receptor activator of nuclear factor$\kappa \mathrm{B}$ ligand (RANKL)-mediated osteoclastogenesis and bone-resorbing activity in vitro and attenuated lipopolysaccharide (LPS)-induced bone destruction in vivo. KP-A038 significantly inhibited the induction of nuclear factor of activated T-cells, cytoplasmic 1 (NFATC1) and the expression of its target genes, including tartrate-resistant acid phosphatase (Acp5), cathepsin $\mathrm{K}$ (Ctsk), dendritic cell-specific transmembrane protein (Dcstamp), and matrix metallopeptidase 9 (Mmp9). KP-A038 upregulated the expression of negative regulators of osteoclast differentiation, such as interferon regulatory factor-8 (IIf8) and B-cell lymphoma 6 (BC/6). Consistently, KP-A038 downregulated the expression of B lymphocyte-induced maturation protein-1 (Blimp1 encoded by Prdm1), a repressor for Irf8 and Bcl6. Moreover, administration of KPA038 reduced LPS-induced bone erosion by suppressing osteoclast formation in vivo. Thus, our findings suggest that KP-A038 may serve as an effective therapeutic agent for the treatment and/or prevention of bone loss in pathological bone diseases, including osteoporosis and periodontitis.

Keywords: imidazole, KP-A038, osteoclast, differentiation, bone resorption 


\section{INTRODUCTION}

Bone remodeling comprises of resorption of aged or damaged tissue by osteoclasts and replacement of new bone by osteoblasts, which is delicately regulated in a steady physiological state. This process occurs continuously to maintain skeletal integrity and strength and mineral homeostasis. Enhanced osteoclast differentiation and resorbing activity lead to loss of bone mass and architectural deterioration of bone tissue, which is mainly responsible for osteolytic bone diseases, including osteoporosis, periodontitis, and rheumatoid arthritis (Manolagas and Jilka, 1995; Sato and Takayanagi, 2006; Bartold et al., 2010). Therefore, various studies have focused on the development of novel treatments targeting osteoclast formation and activation to prevent and treat osteolytic lesions.

Osteoclasts, the only cells capable of degrading the bone matrix, are formed through multiple steps, including proliferation, differentiation of monocyte/macrophage lineage progenitors into mononuclear preosteoclasts, fusion of preosteoclasts, and activation to break down bone (Chambers, 2000; Teitelbaum, 2000). Mature osteoclasts exhibit unique morphological characteristics, including a ruffled border membrane and an actin ring structure (Teitelbaum, 2011). Osteoclast differentiation and function are governed by two essential factors: macrophage-colony stimulating factor (M-CSF), required for the survival and proliferation of progenitor cells, and receptor activator of nuclear factor-kB ligand (RANKL), primarily involved in differentiation to osteoclasts (Feng and Teitelbaum, 2013). Upon binding of RANKL to its receptor RANK, intracellular signaling pathways, such as mitogenactivated protein kinases (MAPKs) and nuclear factor-kappa B (NF-KB), are activated, which eventually lead to the induction of nuclear factor of activated T-cells cytoplasmic 1 (NFATc1), a key regulator of osteoclast differentiation (Wong et al., 1998; Lee and Kim, 2003). The transcriptional activity of NFATc1 is repressed by several negative regulators, such as interferon regulatory factor-8 (IRF-8), v-Maf musculoaponeurotic fibrosarcoma oncogene family member protein B (MafB), and B-cell lymphoma 6 (Bcl6) (Kim et al., 2007; Zhao et al., 2009; Park-Min et al., 2013). During osteoclast differentiation, RANKLinduced B lymphocyte-induced maturation protein-1 (Blimp1, encoded by $\operatorname{Prdm} 1$ ) upregulation acts as a transcriptional repressor of these anti-osteoclastogenic transcription factors (Nishikawa et al., 2010).

Imidazole is a heterocyclic ring compound with molecular formula $\mathrm{C}_{3} \mathrm{H}_{4} \mathrm{~N}_{2}$ and is a major constituent of various biological molecules, such as histidine, vitamin B12, and biotin (De Luca, 2006). Imidazole derivatives have been reported to exhibit a broad spectrum of biological and pharmacological effects, including anti-inflammatory, antiviral, antitumor, antifungal, and antimycobacterial activity, and numerous commercial drugs, like cimetidine, azithromycin, and metronidazole, contain imidazole nucleus in their structure (Fukui et al., 1982; el-Feky and Abd el-Samii, 1995; Johnson et al., 1999; Banfi et al., 2006). Due to these favorable and beneficial activities, studies have focused on the development of imidazole-based drugs in the pharmaceutical field (De Luca, 2006). Previous studies related to bone metabolism have revealed that imidazole and its analogs could suppress bone resorption, and a substituted imidazole, 4-nitroimidazole derivative, could inhibit RANKL-mediated osteoclast differentiation (Heersche and Jez, 1981; Chen et al., 2006). In addition, zoledronic acid, a potent thirdgeneration bisphosphonate, contains an imidazole ring in the side chain (Paiva-Fonseca et al., 2014). However, it can induce unwanted adverse effects ranging from common to rare (Lambrinoudaki et al., 2008).

In order to develop a novel antiresorptive agent, we synthesized a series of imidazole derivatives and investigated their effects on osteoclastogenesis and bone-resorbing activity. In the present study, we demonstrated that KP-A038 significantly suppressed RANKL-mediated osteoclastogenesis in vitro and reduced lipopolysaccharide (LPS)-induced inflammatory bone erosion in vivo.

\section{MATERIALS AND METHODS}

\section{Antibodies and Reagents}

Antibodies against phospho-p38, phospho-JNK, phosphoERK, and phospho-IкB $\alpha$ were purchased from Cell Signaling Technology (Danvers, MA, United States). Anti-NFATc1 antibody was purchased from BD Pharmingen ${ }^{\mathrm{TM}}$ (San Diego, CA, United States), and antibody against IRF8 was obtained from Santa Cruz Biotechnology. Recombinant M-CSF and RANKL were obtained from R\&D Systems (Minneapolis, MN, United States). Fetal bovine serum (FBS) and $\alpha$-minimum essential medium ( $\alpha$-MEM) were obtained from Gibco BRL (Grand Island, NY, United States). KP-A038 is the imidazobenzimidazole compound of in-house chemical library and the chemical name of KP-A038 is (2-([1,1'-biphenyl]-4-yl)-1(2-(piperidin-1-yl)ethyl)-1H-benzo[d]imidazo[1,2-a]imidazole

(Figure 1). KP-A038 was synthesized as described previously (Supplementary Figure S1; Kim et al., 2014), and dissolved in DMSO for further experiments.

\section{In vitro Osteoclast Differentiation}

Mouse bone marrow cells isolated from the tibiae and femora of 6-8 week-old male C57/B6L mice (Dae Han Bio

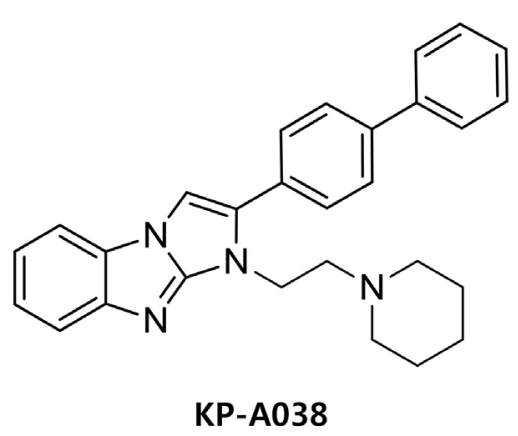

FIGURE 1 | Chemical structures of KP-A038. 
Link Co., LTD., Chungbuk, South Korea) were incubated in $\alpha$-MEM supplemented with 10\% FBS (Ihn et al., 2017; Yoon et al., 2018). After 24 h, non-adherent cells were collected and cultured in $\alpha$-MEM containing 10\% FBS and M-CSF (30 ng/mL) for 3 days to generate bone marrowderived macrophages (BMMs). BMMs were plated in 96-well plates and incubated in osteoclast-inducing media containing $20 \mathrm{ng} / \mathrm{mL}$ RANKL and $10 \mathrm{ng} / \mathrm{mL} \mathrm{M}-\mathrm{CSF}$ with or without various concentrations of KP-A038. The media were changed every 2 days until mature osteoclasts were formed. The cells were fixed in $4 \%$ paraformaldehyde, and formation of osteoclasts was determined using an Acid Phosphatase, Leukocyte (TRAP) staining kit (Sigma-Aldrich, St. Louis, MO, United States). TRAP-positive multinucleated cells (MNCs) with more than three nuclei were scored as osteoclastlike cells.

\section{Cell Viability Assay}

The cytotoxic effect of KP-A038 on cell viability was determined using Cell Counting Kit-8 (CCK-8; Dojindo Molecular Technologies Inc., Rockville, MD, United States) following the manufacturer's instructions. BMMs seeded in 96-well plates $\left(3 \times 10^{3}\right.$ cells/well $)$ were cultured in $\alpha$-MEM containing $10 \%$ FBS and M-CSF (10 ng/mL) in the presence or absence of various doses of KP-A038 for 3 days. The media were replaced with fresh medium containing $10 \% \mathrm{CCK}-8$, and the cells were incubated at $37^{\circ} \mathrm{C}$ for $2 \mathrm{~h}$. The absorbance at $450 \mathrm{~nm}$ was measured using a 96-well microplate reader (Bio-Rad Laboratories, Hercules, CA, United States).

\section{Quantitative Real-Time PCR}

Bone marrow-derived macrophages were cultured in 6-well plates with or without $5 \mu \mathrm{M}$ KP-A038 in osteoclastinducing media. Total RNA was extracted using TRI-solution (Bioscience, Seoul, South Korea) according to the manufacturer's instructions. cDNA was synthesized using SuperScript II Reverse Transcriptase (Invitrogen, Carlsbad, CA, United States). Realtime PCR was performed using a LightCycler 1.5 real-time PCR system (Roche Diagnostics, Basel, Switzerland) and the SYBR Premix Ex Taq (Takara Bio Inc., Shiga, Japan) (Ihn et al., 2018). The primer sequences used in real-time PCR analysis were: Acp5, 5'-TCCCCAATGCCCCATTC- $3^{\prime}$ and $5^{\prime}$ CGGTTCTGGCGATCTCTTTG-3'; Ctsk， 5'-GGCTGTGGAG GCGGCTAT-3' and 5'-AGAGTCAATGCCTCCGTTCTG3'; Mmp9, 5'-AAAGACCTGAAAACCTCCAACCT-3' and $5^{\prime}$-GCCCGGGTGTAACCATAGC-3'; Dcstamp, 5'-CTTC CGTGGGCCAGAAGTT-3' and 5'-AGGCCAGTGC TGACTAGGATGA-3'; Nfatc1, 5' -ACCACCTTTCCGCAACCA-

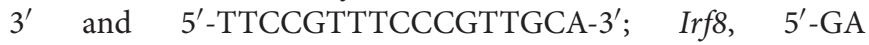
TCGAACAGATCGACAGCA- $3^{\prime}$ and $5^{\prime}$-AGCACAGCGTAA CCTCGTCT-3'; Bcl6, 5'-ATGAGATTGCCCTGCATTTC-3' and $5^{\prime}$-TTCTTCCAGTTGCAGGCTTT-3'; Ifng, $5^{\prime}$-TCAAGT GGCATAGATGTGGAAGAA- $3^{\prime}$ and $5^{\prime}$-TGGCTCTGCAGGATT TTCATG-3'; Prdm 1, 5'-TTCTTGTGTGGTATTGTCGGG

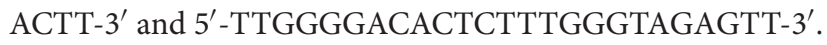

\section{Immunoblotting}

Cells were lysed with RIPA buffer containing protease and phosphatase inhibitors. Equal volume of cell lysates $(25 \mu \mathrm{g}$ of protein) was loaded onto $10 \%$ sodium dodecyl sulfatepolyacrylamide gels, followed by transfer to nitrocellulose membranes (Whatman Inc., Florham Park, NJ, United States). The membranes were placed in a blocking solution [3\% non-fat dry milk in TBS-T (25 mM Tris-HCl, pH 7.4, $150 \mathrm{mM} \mathrm{NaCl}$, and $0.2 \%$ Tween 20)] for $1 \mathrm{~h}$. After blocking, the membranes were incubated with specific primary antibodies $(1: 1000)$ at $4^{\circ} \mathrm{C}$ overnight. Following incubation with secondary antibodies, protein signals were detected using WesternBright ECL (Advansta, Menlo Park, CA, United States), and imaged with an X-ray film or by using chemiluminescence imager (Azure Biosystems, Inc., Dublin, CA, Unites States).

\section{Staining of Actin Rings}

Bone marrow-derived macrophages grown on glass coverslips were cultured with $10 \mathrm{ng} / \mathrm{mL}$ M-CSF and 20 $\mathrm{ng} / \mathrm{mL}$ RANKL in the presence or absence of $5 \mu \mathrm{M} \mathrm{KP}$ A038. The cells were washed with phosphate buffered saline, fixed with $4 \%$ paraformaldehyde, and permeabilized with $0.1 \%$ Triton X-100. F-actin was stained with rhodamine conjugated phalloidin (Cytoskeleton, Denver, CO, United States), and 4,6-diamidino-2-phenylindole dihydrochloride (Santa Cruz Biotechnology, Santa Cruz, CA, United States) was used for nuclei staining. The images were acquired using a BX51 Fluorescent Microscope (Olympus, Tokyo, Japan).

\section{Resorption Pit Assay}

Bone marrow-derived macrophages were placed on bone slices (IDS Nordic Bioscience, Herlev, Denmark) and incubated with M-CSF (10 ng/mL) and RANKL (20 ng/mL) to induce differentiation of BMMs into multinucleated osteoclasts. After 3 days, the cells were treated with vehicle or $5 \mu \mathrm{M} \mathrm{KP}$ A038 for 2 days. The cells removed with $1 \mathrm{~N} \mathrm{NaOH}$ for $20 \mathrm{~min}$, followed by staining with hematoxylin to identify the areas of resorption pits. The resorbed areas were measured using the i-Solution image analysis program (IMT i-Solution, Daejeon, South Korea).

\section{In vivo LPS-Induced Bone Loss}

All animal experiments were approved by the Animal Care and Use Committee at Kyungpook National University and were conducted in accordance with the guidelines for the care and use of laboratory animals. To study the effect of KP-A038 on in vivo bone destruction, 8-week-old C57/B6L mice were intraperitoneally injected with KP-A038 (30 $\mathrm{mg} / \mathrm{kg}$ ) or vehicle daily for 9 days, and LPS $(5 \mathrm{mg} / \mathrm{kg}$ ) was intraperitoneally administered to the mice on days 2 and 6, as previously described (Ihn et al., 2015a). On day 10 , the mice were euthanized by cervical dislocation under anesthesia with avertin. The femurs were isolated and fixed in $4 \%$ paraformaldehyde for $18 \mathrm{~h}$. 

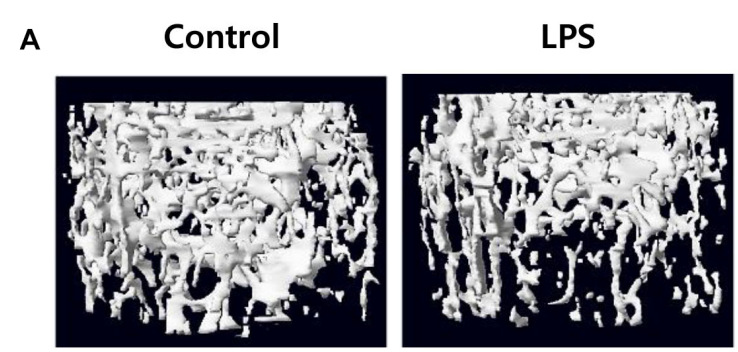

LPS+KP-A038
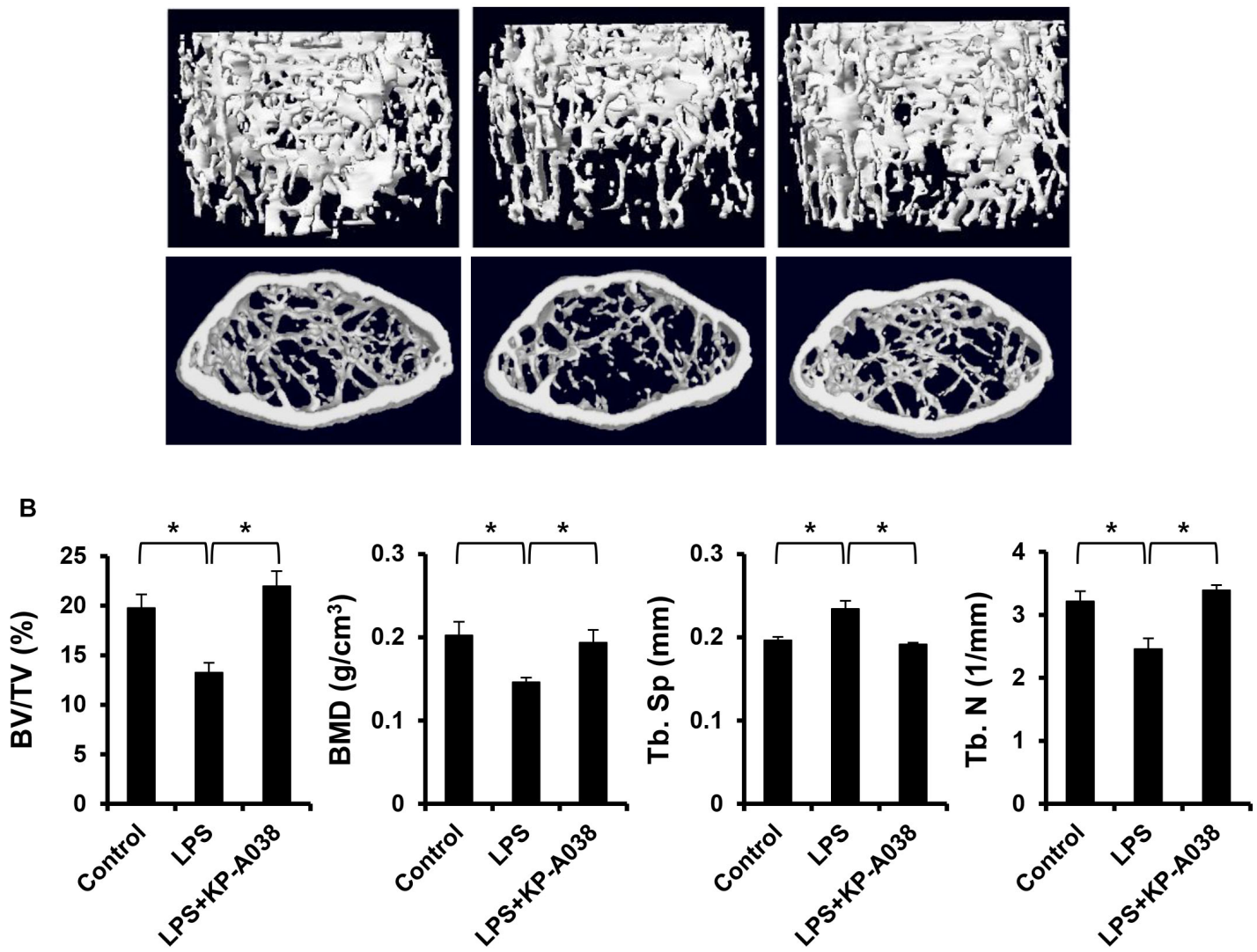

FIGURE 2 | KP-A038 mitigated femoral bone loss induced by LPS in vivo. (A) Representative micro-CT images of distal femurs of three groups. (B) Quantification of bone volume per tissue volume (BV/TV), bone mineral density (BMD), trabecular separation (Tb. Sp), and trabecular number (Tb. N) from each group. $n=5$ (10 legs) in each group. ${ }^{*} p<0.05$.

\section{Micro-CT and Histomorphometric Analysis}

The fixed femurs were scanned using high-resolution $\mu \mathrm{CT}$ (Skyscan 1272; Kontich, Belgium) with a source voltage of $60 \mathrm{kV}$, current of $166 \mu \mathrm{A}$, and resolution of $14 \mu \mathrm{m}$. Bone morphometric parameters, including bone volume per total volume (BV/TV), bone mineral density (BMD), trabecular separation (Tb. Sp.), and trabecular number (Tb. N.) were analyzed using CTAn software (Bruker; Kontich, Belgium). Micro-CT 3D images of trabecular bones in the distal femurs were generated using CTAn/CTVol software (Bruker; Kontich, Belgium).

For histology, the fixed femurs were decalcified in 12\% EDTA and embedded in paraffin, and the histological tissue sections were stained with hematoxylin and eosin and TRAP.

\section{Statistics}

Experiments were conducted three times, and all data are presented as mean \pm standard deviation (SD). Statistical significance was evaluated by the two-tailed Student's $t$-test or one-way analysis of variance (ANOVA) with Tukey's multiple comparison post hoc test. $p<0.05$ or $p<0.01$ was considered statistically significant.

\section{RESULTS}

\section{KP-A038 Prevents LPS-Induced Bone Resorption in vivo}

To examine the in vivo efficacy of KP-A038 in osteoclast formation and bone resorption, we used an LPS-induced bone erosion model. Mice were intraperitoneally administered with LPS on days 2 and 6 and received vehicle or KP-A038 daily for 9 days. Injection of KP-A038 alone did not cause adverse events, including death, abnormal behavior, sickness, and distress or change bone parameters (Supplementary Figure S2). Administration of LPS led to a reduction in trabecular bone mass of femurs, and this LPS-mediated bone loss was suppressed by co-injection with KP-A038 (Figure 2A). Three-dimensional morphometric analysis of distal femurs showed that BV/TV, $\mathrm{BMD}$, and $\mathrm{Tb}$. N were significantly reduced in LPS-treated group (Figure 2B). Such reduction in bone parameters was attenuated by KP-A038 treatment (Figure 2B). In correlation with $\mu \mathrm{CT}$ images and analysis of bone parameters, the histological sections stained with hematoxylin and eosin or TRAP showed that KPA038 effectively suppressed LPS-induced osteoclast formation and subsequent bone loss in vivo (Figure 3). 

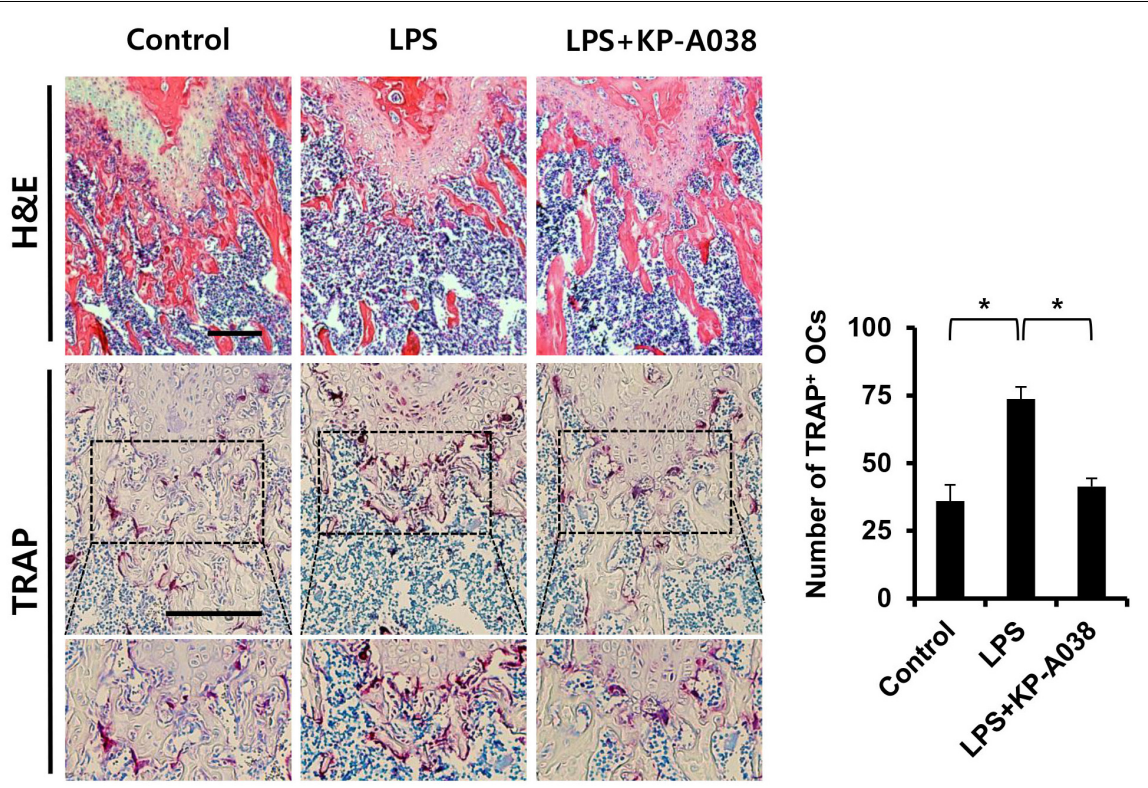

FIGURE 3 | Histological analysis of the effect of KP-A038 on LPS-mediated bone destruction. Fixed femurs were decalcified and sectioned. The sections were stained with hematoxylin and eosin (upper panel) and tartrate-resistant acid phosphatase (TRAP, middle and lower panel). The number of osteoclasts was analyzed in the TRAP-stained slices (right graph). $n=5$ (10 legs) in each group. ${ }^{*} p<0.05$.

A
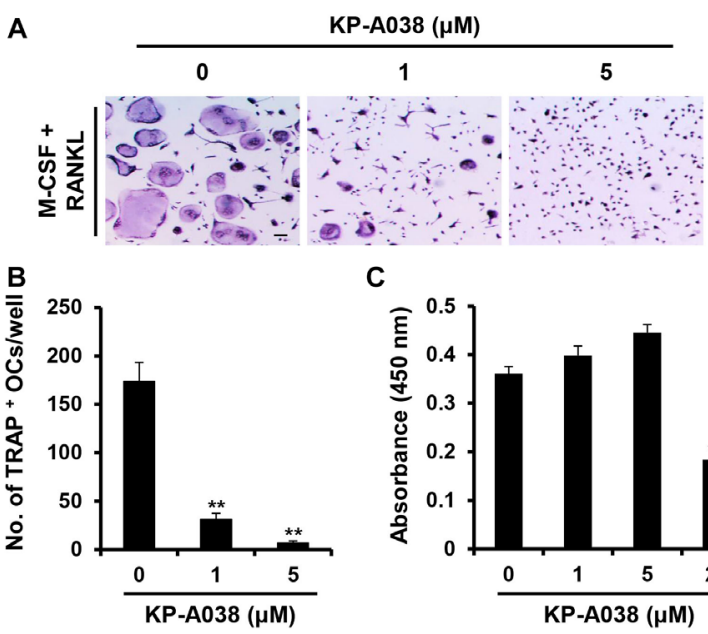

C

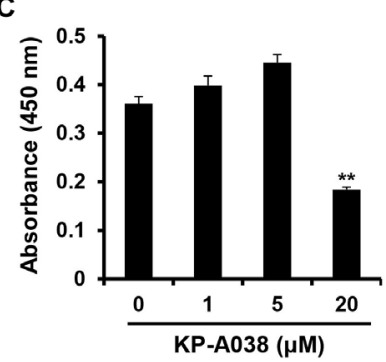

FIGURE 4 | KP-A038 inhibited RANKL-mediated osteoclastogenesis without cytotoxicity. (A) BMMs were incubated in osteoclastogenic medium supplemented with M-CSF $(10 \mathrm{ng} / \mathrm{mL})$ and RANKL $(20 \mathrm{ng} / \mathrm{mL})$ in the presence of KP-A038 or vehicle. The cells were stained to evaluate TRAP activity. (B) The number of osteoclast-like cells was quantified. (C) BMMs were cultured with M-CSF (10 ng/mL) and various doses of KP-A038 for 3 days. Cell viability was assessed by CCK-8 assay. ${ }^{* *} p<0.01$.

\section{KP-A038 Inhibits RANKL-Induced Osteoclast Formation in vitro}

To clarify whether KP-A038 affects the viability of osteoclast progenitors, CCK- 8 assay was performed using primary BMMs cultured with M-CSF and various doses of KP-A038 for 3 days. As shown in Figure 4C, KP-A038 at concentrations of up to $5 \mu \mathrm{M}$ did not decrease the rate of proliferation and survival of BMMs. Hence, $5 \mu \mathrm{M}$ KP-A038 was chosen for subsequent in vitro studies. As primary BMMs are capable of differentiating into multinucleated osteoclasts in response to M-CSF and RANKL, we first examined the effect of KP-A038 on osteoclast formation in BMMs. Primary cultured BMMs were treated with KP-A038 $(1 \mu \mathrm{M}$ or $5 \mu \mathrm{M})$ in osteoclastinducing media supplemented with M-CSF and RANKL. RANKL promoted the formation of osteoclasts (TRAP-positive MNCs) from progenitors (BMMs) in the vehicle-treated control group, whereas treatment with KP-A038 markedly suppressed osteoclast formation in a concentration-dependent manner (Figure 4A). In the presence of $5 \mu \mathrm{M} \mathrm{KP}$-A038, the number of TRAP-positive MNCs was reduced by $96 \%$ (Figure 4B).

\section{KP-A038 Attenuates the Expression of Osteoclast-Specific Markers as Well as the Formation of Actin Rings}

We next evaluated the mRNA and protein expression levels of osteoclast-specific markers during RANKL-induced osteoclast differentiation via real-time PCR and western blotting to further determine its inhibitory role in osteoclastogenesis. Consistent with the decreased osteoclast formation, treatment with KPA038 $(5 \mu \mathrm{M})$ downregulated the mRNA levels of Acp5, Ctsk, Dcstamp, Mmp9, and Nfatcl, which are required for osteoclast formation and/or bone resorption (Figures 5A,B). In addition, the induction of both cathepsin K (Ctsk) and NFATc1 proteins and the nuclear expression of NFATc1 were decreased by treatment with $5 \mu \mathrm{M}$ KP-A038 (Figures 5C,F).

An essential step in the generation of multinucleated osteoclasts is cell-cell fusion, and DC-STAMP is a key regulator 

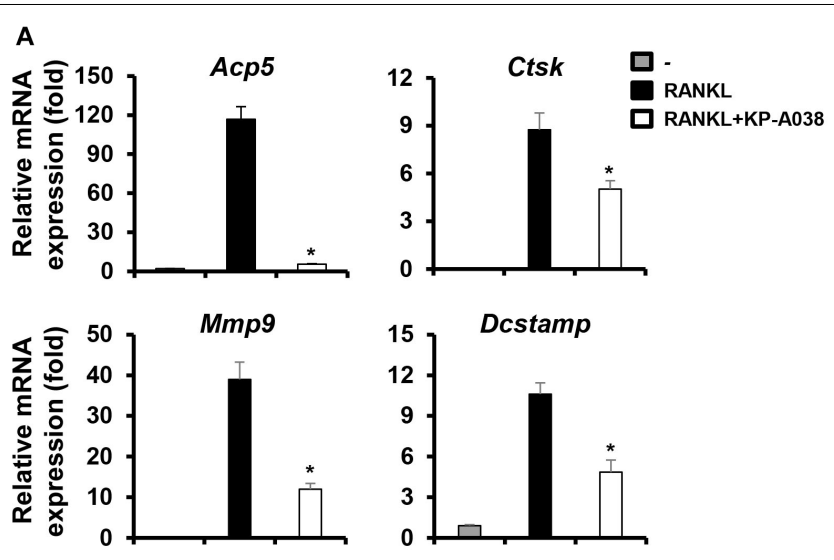

B $\square$ -

RANKL

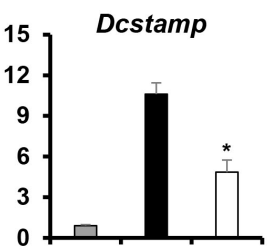

\section{$\square$ RANKL+KP}

C
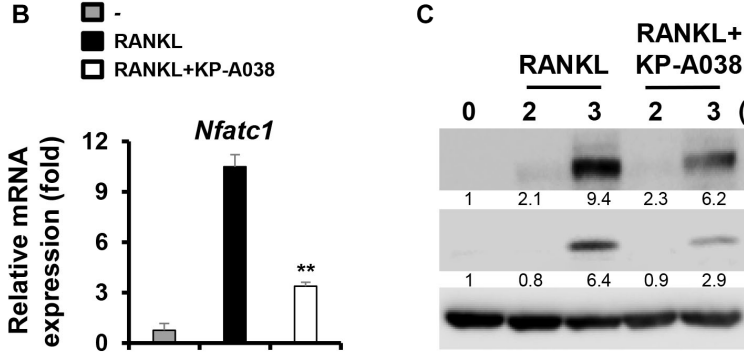

$\begin{array}{llll} & \text { RANKL } & \frac{\text { KP-A038 }}{23} & 3 \text { (days) }\end{array}$

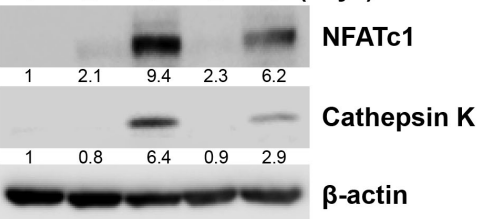

D
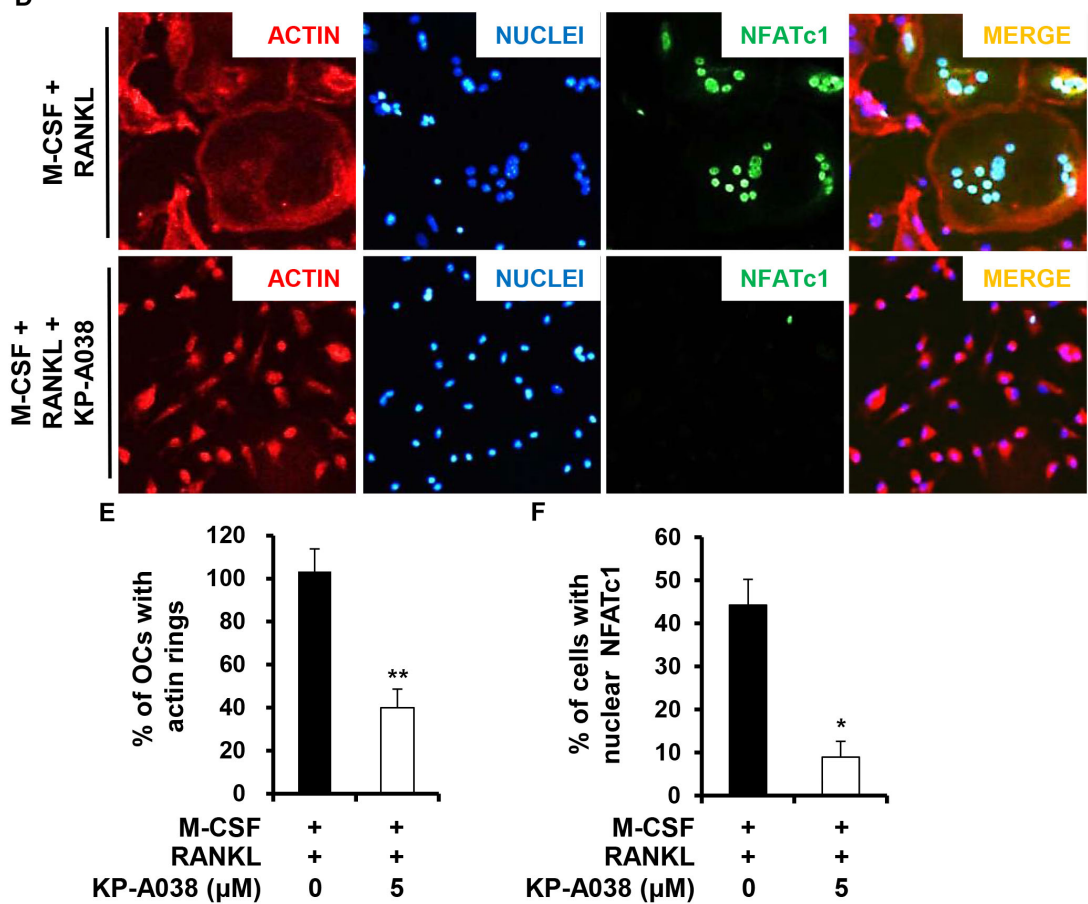

FIGURE 5 | KP-A038 suppressed the expression of osteoclast-specific markers and the formation of actin rings. (A-C) BMMs were incubated in osteoclastogenic medium with KP-A038 $(5 \mu \mathrm{M})$ or vehicle for the indicated times. The mRNA (A,B) and protein $\mathbf{( C )}$ expression levels of markers were evaluated by real-time PCR and western blot analysis, respectively. (D) BMMs were seeded on glass coverslips and cultured with M-CSF (10 ng/mL) and RANKL (20 ng/mL) in the presence or absence of $5 \mu \mathrm{M} \mathrm{KP-A038.} \mathrm{Cells} \mathrm{were} \mathrm{fixed,} \mathrm{and} \mathrm{F-actin} \mathrm{structures} \mathrm{and} \mathrm{nuclei} \mathrm{were} \mathrm{visualized} \mathrm{by} \mathrm{staining} \mathrm{with} \mathrm{rhodamine-conjugated} \mathrm{phalloidin} \mathrm{and}$

4',6-diamidino-2-phenylindole dihydrochloride, respectively. Quantification of the percentage of (E) osteoclasts with actin rings and (F) cells with nuclear NFATc1. $* p<0.05, * * p<0.01$ 
of this process (Yagi et al., 2005). In accordance with the decreased induction of Dcstamp, we found that the number of multinucleated giant cells with actin ring structure was significantly decreased by KP-A038 treatment (Figures 5D,E).

\section{KP-A038 Impairs the Bone-Resorbing Function of Osteoclasts}

We next examined if KP-A038 affects the bone-resorbing activity of osteoclasts. BMMs were plated on bone slices in osteoclastogenic media to generate multinucleated osteoclast-like cells. Then, the cells were treated with vehicle or $5 \mu \mathrm{M} \mathrm{KP}-\mathrm{A} 038$ in osteoclast-inducing media for 2 days. Analysis of resorption pit showed that KP-A038 treatment significantly reduced the formation of resorption pits compared to vehicle treatment, which resulted in formation of larger resorbed areas. Addition of KP-A038 resulted in an $87 \%$ reduction of the resorbed area (Figure 6A), thereby suggesting that KP-A038 directly attenuates the bone-resorbing function of osteoclasts.

\section{KP-A038 Inhibits the Repression of Negative Regulators of Osteoclast Differentiation}

To understand the molecular mechanism of the inhibitory action of KP-A038 in osteoclast differentiation and function, the effect

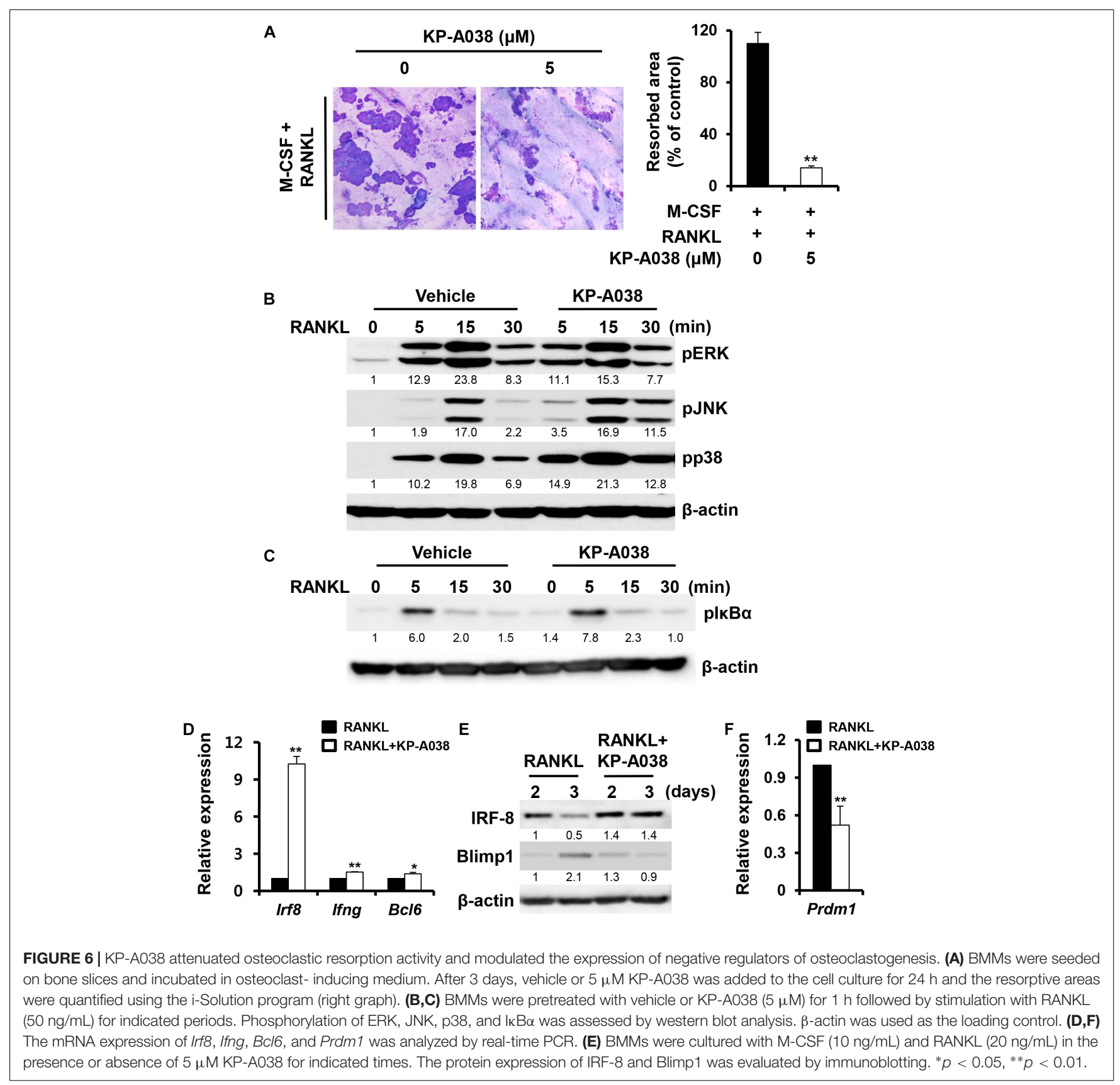


of KP-A038 on RANKL-stimulated MAPK and NF- $\mathrm{B}$ signaling pathways was examined. Serum-starved BMMs were stimulated with RANKL after pretreatment with vehicle or $5 \mu \mathrm{M}$ KP-A038 for $1 \mathrm{~h}$. Stimulation with RANKL led to activation of MAPKs (ERK, p38, and JNK) and IKB $\alpha$ within $15 \mathrm{~min}$ in control cells, and pretreatment with KP-A038 had no effect on the phosphorylation levels of MAPKs and IKB $\alpha$, indicating that KP-A038 does not affect the MAPK and NF- $\kappa$ B signaling pathways (Figures 6B,C).

RANKL-RANK signaling pathways ultimately converge on the induction of NFATc1, which is the main transcriptional regulator of osteoclastogenesis, and its activity is negatively regulated by IRF- 8 and Bcl6 during osteoclast differentiation (Zhao et al., 2009; Miyauchi et al., 2010). Hence, we examined the expression levels of anti-osteoclastogenic genes, such as Irf8, Ifng, and Bcl6. While the mRNA expression of Irf8, Ifng, and Bcl6 was downregulated by RANKL stimulation in vehicletreated control group, such downregulation was blocked in the presence of $5 \mu \mathrm{M}$ KP-A038 (Figure 6D). As shown in Figure 6E, IRF-8 protein expression was similarly upregulated in a time-dependent manner. Blimp1 (encoded by $\operatorname{Prdm} 1$ ) is known to function as a transcriptional repressor of IRF-8 and Bcl6, and Blimp1-mediated Irf8, and Bcl6 suppression is critical for osteoclast differentiation (Zhao et al., 2009; Miyauchi et al., 2010). Consistent with increased Irf8 and Bcl6 expression, Blimp1 mRNA and protein levels were reduced in the presence of KPA038 (Figures 6E,F).

\section{DISCUSSION}

Although the bone is remodeled through coordinated destruction and deposition, and the remodeling process is strictly regulated, abnormal bone remodeling can arise due to various causes, inducing skeletal diseases ranging from mild to severe. In particular, enhanced osteoclastogenesis and resorbing activity of mature osteoclasts causes destructive bone diseases. Therefore, the suppression of osteoclast differentiation and bone-resorbing function of mature osteoclasts is an important aspect for preventing and treating osteolytic diseases. In the current study, we observed that KP-A038, a novel imidazole compound, possessed anti-osteoclastogenic activity via downregulation of NFATc1 and modulation of the expression of negative regulators of osteoclast differentiation. Consistent with the suppression of osteoclast differentiation in vitro, results from the in vivo LPS-induced bone destruction study demonstrated that KP-A038 protected against bone loss by attenuation of osteoclast formation.

RANKL-RANK signaling ultimately causes the induction of NFATc1, which acts as a key transcription factor of osteoclastogenesis. Even though RANKL signaling is required for osteoclast differentiation, embryonic stem cells lacking NFATc1 fail to undergo osteoclast differentiation even in the presence of RANKL (Takayanagi et al., 2002). Furthermore, ectopic expression of NFATc1 in BMMs leads to osteoclast differentiation without RANKL signaling (Takayanagi et al., 2002). Overexpression of NFATc1 in osteoclast precursors lacking c-Fos, a pivotal regulator of early activation of NFATc1, rescues osteoclast differentiation in vitro (Matsuo et al., 2004). Aliprantis et al. (2008) reported that osteoclast-specific NFATc1 deficiency causes osteopetrosis due to impaired osteoclast differentiation. These in vivo and in vitro studies have established the essential role of NFATc1 in osteoclast formation and function. We demonstrated that KP-A038 treatment markedly downregulated both mRNA and protein levels of NFATc1, resulting in inhibition of osteoclastogenesis (Figures 5B,C). As expected, downregulation of NFATc1 by KP-A038 treatment attenuated the expression of direct transcriptional target genes, including Acp5, Ctsk, Dcstamp, and Mmp9 (Figure 5A). Among them, DC-STAMP is essential for cell-to-cell fusion, which is a critical process to generate MNCs and reorganize the actin cytoskeleton during osteoclast differentiation (Yagi et al., 2005). Due to the reduced Dcstamp levels, KP-A038 treatment suppressed the formation of actin rings (Figure 5D). Our study suggested that KP-A038 might target NFATc1 leading to inhibition of osteoclast differentiation and impairment of cellular fusion.

The activation of signaling molecules, like MAPKs and NF- $\mathrm{BB}$ involved in the RANKL/RANK signaling pathway, is an early cellular event of osteoclast differentiation, eventually leading to the induction NFATc1 (Iotsova et al., 1997; Mizukami et al., 2002). To understand the molecular mechanisms of inhibition of osteoclast differentiation and bone-resorbing function, we analyzed the extent of phosphorylation of the signaling molecules. Although our studies showed that KP-A038 definitely reduced the expression of NFATc1 and its target genes, KP-A038 treatment did not inhibit the activation of RANKLmediated MAPKs and NF- $\kappa B$ (Figures 6B,C). Previously we screened and identified several chemicals from our in-house chemical library that exerted inhibitory effects on osteoclast differentiation and bone resorption. Although those compounds exhibited anti-osteoclastogenic and antiresorptive activities with a similar inhibitory concentration, the inhibitory mechanism and chemical structure of the compounds were quite distinct one another (Ihn et al., 2015a,b, 2017, 2018). In particular, unlike our previous compounds, KP-A038 did not inhibit an early RANKL-RANK signaling pathways (Figures 6B,C), suggesting different mode of action of KP-A038 from our previous chemicals. RANKL/RANK signaling has also been shown to downregulate various transcriptional repressors, including MafB, IRF-8, and Bcl6, which act as anti-osteoclastogenic factors via downregulating NFATc1 expression (Kim et al., 2007; Zhao et al., 2009; Miyauchi et al., 2010). Nishikawa et al. found that a transcriptional repressor of the negative regulators of NFATc1, Blimp 1 (Prdm1), is induced by RANKL stimulation, and Blimp 1-mediated suppression of anti-osteoclastogenic factors is necessary for osteoclastogenesis (Nishikawa et al., 2010). Therefore, forced expression of the negative regulators leads to impaired osteoclast differentiation. Among them, IRF8 suppresses NFATc1 autoamplification and its transcriptional activity, and Bcl6 inhibits the expression of NFATc1 and its target genes associated with osteoclastogenesis and bone resorption (Zhao and Ivashkiv, 2011). In our study, KP-A038 prevented the downregulation of negative regulators of osteoclastogenesis, and in particular, the mRNA and protein expression of IRF-8 was strongly induced in the presence of KP-A038 
(Figures 6D,E). Consistently, KP-A038 suppressed both mRNA and protein levels of Blimp1 (Prdm1) (Figures 6E,F). These results indicated that the inhibitory effect of KP-A038 on osteoclastogenesis might be in part mediated by the failure of repression of negative regulators. Further studies would be necessary to identify the primary target (s) for KP-A038.

The major role of osteoclasts is to break down the bone matrix, which is termed as bone resorption. Various antiresorptive drugs, such as bisphosphonates and selective estrogen receptor modulators have been clinically used for preventing further loss of bone density (Migliaccio et al., 2007). Therefore, we evaluated the effect of KP-A038 on bone-resorbing function by resorption pit assay. KP-A038, at a concentration that inhibited osteoclast differentiation, considerably suppressed the ability of osteoclasts to degrade the calcified bone matrix (Figure 6A), indicating that KP-A038 exhibits not only anti-osteoclastogenic but also antiresorptive activity.

Lipopolysaccharide is a structural constituent of the outer membrane of gram-negative bacteria and it functions as a potent stimulator of bone loss. The production of inflammatory cytokines in response to LPS is increased, which directly and indirectly contributes to stimulation of osteoclastogenesis and inflammatory bone loss (Wada et al., 2004; Islam et al., 2007). Especially, alveolar bone destruction mediated by inflammatory responses in the oral cavity can lead to tooth loss (Pihlstrom et al., 2005). For that reason, it is critical to prevent the balance of two opposing activities (bone formation and bone resorption) from breaking for maintenance and regeneration of alveolar bone and supporting tissue (Larsson et al., 2016). Consistent with its inhibitory effect on osteoclast differentiation and function in vitro, KP-A038 attenuated the femoral bone destruction induced by LPS (Figure 2). Administration of KPA038 decreased the numbers of TRAP-positive osteoclasts as well as bone resorption in vivo (Figure 3), indicating that KPA038 may have the potential to not only protect from the risk of inflammatory bone loss in periodontitis but also to contribute to bone regeneration in the oral cavity.

\section{CONCLUSION}

Developing alternative agents that modulate excessive osteoclast formation and bone resorption is an important and urgent

\section{REFERENCES}

Aliprantis, A. O., Ueki, Y., Sulyanto, R., Park, A., Sigrist, K. S., Sharma, S. M., et al. (2008). NFATc1 in mice represses osteoprotegerin during osteoclastogenesis and dissociates systemic osteopenia from inflammation in cherubism. J. Clin. Invest. 118, 3775-3789. doi: 10.1172/ JCI35711

Banfi, E., Scialino, G., Zampieri, D., Mamolo, M. G., Vio, L., Ferrone, M., et al. (2006). Antifungal and antimycobacterial activity of new imidazole and triazole derivatives. A combined experimental and computational approach. J. Antimicrob. Chemother. 58, 76-84. doi: 10.1093/jac/ dkl182

Bartold, P. M., Cantley, M. D., and Haynes, D. R. (2010). Mechanisms and control of pathologic bone loss in periodontitis. Periodontology 2000, 55-69. doi: $10.1111 /$ j.1600-0757.2010.00347.x task. The results of this study demonstrated that KP-A038 exhibited anti-osteoclastogenic and antiresorptive properties by inhibiting the induction of NFATc1 via modulating the expression of negative regulators of osteoclastogenesis. Furthermore, KP-A038 protected against LPS-induced femoral bone loss in vivo. Our results suggest that KPA038 might serve as a novel antiresorptive agent for osteoclast-related diseases.

\section{ETHICS STATEMENT}

All animal experiments were approved by the Animal Care and Use Committee at Kyungpook National University and were conducted in accordance with the guidelines for the care and use of laboratory animals.

\section{AUTHOR CONTRIBUTIONS}

HI, TL, and EP designed the research and wrote the manuscript. HI and DL performed the research and collected the data. J-SB, S-HK, IJ, YB, and H-IS analyzed the data and clarified the manuscript.

\section{FUNDING}

This work was supported by the National Research Foundation of Korea (NRF) grant funded by the Korean government (MSIT; NRF-2017R1A2B1005409 and NRF-2017R1A5A2015391) and the Bio \& Medical Technology Development Program of the NRF, which was funded by the Government of South Korea (MSIT; NRF-2017M3A9E4047244).

\section{SUPPLEMENTARY MATERIAL}

The Supplementary Material for this article can be found online at: https://www.frontiersin.org/articles/10.3389/fphar. 2019.00367/full\#supplementary-material

Chambers, T. J. (2000). Regulation of the differentiation and function of osteoclasts. J. Pathol. 192, 4-13. doi: 10.1002/1096-9896(2000)9999:9999<:: AID-PATH645>3.0.CO;2-Q

Chen, T., Knapp, A. C., Wu, Y., Huang, J., Lynch, J. S., Dickson, J. K., et al. (2006). High throughput screening identified a substituted imidazole as a novel RANK pathway-selective osteoclastogenesis inhibitor. Assay Drug Dev. Technol. 4, 387-396. doi: 10.1089/adt.2006.4.387

De Luca, L. (2006). Naturally occurring and synthetic imidazoles: their chemistry and their biological activities. Curr. Med. Chem. 13, 1-23.

el-Feky, S. A., and Abd el-Samii, Z. K. (1995). Synthesis and antiinflammatory properties of some novel thiazolidinones and imidazolidinones derived from 4-(3-phenyl-4(3H)-quinazolinon-2-yl)-3-thiosemicarbazone. Pharmazie 50, 341-343. doi: 10.1002/chin.199537188

Feng, X., and Teitelbaum, S. L. (2013). Osteoclasts: new insights. Bone Res. 1, 11-26. doi: $10.4248 /$ BR201301003 
Fukui, M., Inaba, M., Tsukagoshi, S., and Sakurai, Y. (1982). New antitumor imidazole derivative, 5-carbamoyl-1H-imidazol-4-yl piperonylate, as an inhibitor of purine synthesis and its activation by adenine phosphoribosyltransferase. Cancer Res. 42, 1098-1102.

Heersche, J. N., and Jez, D. H. (1981). The effect of imidazole-analogues on bone resorption in vitro: a suggested role for thromboxane A2. Prostaglandins 21, 401-411. doi: 10.1016/0090-6980(81)90085-X

Ihn, H. J., Lee, D., Lee, T., Kim, S. H., Shin, H. I., Bae, Y. C., et al. (2015a). Inhibitory effects of KP-A159, a thiazolopyridine derivative, on osteoclast differentiation, function, and inflammatory bone loss via suppression of RANKL-induced MAP kinase signaling pathway. PLoS One 10:e142201. doi: 10.1371/journal.pone. 0142201

Ihn, H. J., Lee, D., Lee, T., Shin, H. I., Bae, Y. C., Kim, S. H., et al. (2015b). The 1,2,3triazole derivative KP-A021 suppresses osteoclast differentiation and function by inhibiting RANKL-mediated MEK-ERK signaling pathway. Exp. Biol. Med. 240, 1690-1697. doi: 10.1177/1535370215576310

Ihn, H. J., Lee, T., Kim, J. A., Lee, D., Kim, N. D., Shin, H. I., et al. (2017). OCLI023 , a novel pyrimidine compound, suppresses osteoclastogenesis in vitro and alveolar bone resorption in vivo. PLoS One 12:e0170159. doi: 10.1371/journal. pone. 0170159

Ihn, H. J., Lee, T., Lee, D., Kim, J. A., Kim, K., Lim, S., et al. (2018). A novel benzamide derivative protects ligature-induced alveolar bone erosion by inhibiting NFATc1-mediated osteoclastogenesis. Toxicol. Appl. Pharmacol. 355, 9-17. doi: 10.1016/j.taap.2018.06.017

Iotsova, V., Caamano, J., Loy, J., Yang, Y., Lewin, A., and Bravo, R. (1997). Osteopetrosis in mice lacking NF-kappaB1 and NF-kappaB2. Nat. Med. 3, 1285-1289. doi: 10.1038/nm1197-1285

Islam, S., Hassan, F., Tumurkhuu, G., Dagvadorj, J., Koide, N., Naiki, Y., et al. (2007). Bacterial lipopolysaccharide induces osteoclast formation in RAW 264.7 macrophage cells. Biochem. Biophys. Res. Commun. 360, 346-351. doi: 10.1016/ j.bbrc.2007.06.023

Johnson, R. A., Huong, S. M., and Huang, E. S. (1999). Inhibitory effect of 4-(4-fluorophenyl)-2-(4-hydroxyphenyl)-5-(4-pyridyl)1H - imidazole on HCMV DNA replication and permissive infection. Antiviral Res. 41, 101-111. doi: 10.1016/S0166-3542(99)00002-9

Kim, K., Kim, J. H., Lee, J., Jin, H. M., Kook, H., Kim, K. K., et al. (2007). MafB negatively regulates RANKL-mediated osteoclast differentiation. Blood 109, 3253-3259. doi: 10.1182/blood-2006-09-048249

Kim, S. Y., Lee, S. H., Shin, J. S., Lee, D., Lee, T., Park, K. C., et al. (2014). A derivative of imidazobenzimidazole, ML106, inhibits melanin synthesis via p38 MAPK activation. Pharmazie 69, 353-357. doi: 10.1691/ph.2014.3868

Lambrinoudaki, I., Vlachou, S., Galapi, F., Papadimitriou, D., and Papadias, K. (2008). Once-yearly zoledronic acid in the prevention of osteoporotic bone fractures in postmenopausal women. Clin. Interv. Aging 3, 445-451. doi: 10.2147/CIA.S2046

Larsson, L., Decker, A. M., Nibali, L., Pilipchuk, S. P., Berglundh, T., and Giannobile, W. V. (2016). Regenerative medicine for periodontal and periimplant diseases. J. Dent. Res. 95, 255-266. doi: 10.1177/0022034515618887

Lee, Z. H., and Kim, H. H. (2003). Signal transduction by receptor activator of nuclear factor kappa B in osteoclasts. Biochem. Biophys. Res. Commun. 305, 211-214. doi: 10.1016/S0006-291X(03)00695-8

Manolagas, S. C., and Jilka, R. L. (1995). Bone marrow, cytokines, and bone remodeling. Emerging insights into the pathophysiology of osteoporosis. N. Engl. J. Med. 332, 305-311. doi: 10.1056/NEJM199502023320506

Matsuo, K., Galson, D. L., Zhao, C., Peng, L., Laplace, C., Wang, K. Z., et al. (2004). Nuclear factor of activated T-cells (NFAT) rescues osteoclastogenesis in precursors lacking c-Fos. J. Biol. Chem. 279, 26475-26480. doi: 10.1074/jbc. M313973200

Migliaccio, S., Brama, M., and Spera, G. (2007). The differential effects of bisphosphonates, SERMS (selective estrogen receptor modulators), and parathyroid hormone on bone remodeling in osteoporosis. Clin. Interv. Aging 2, 55-64. doi: 10.2147/ciia.2007.2.1.55

Miyauchi, Y., Ninomiya, K., Miyamoto, H., Sakamoto, A., Iwasaki, R., Hoshi, H., et al. (2010). The Blimp1-Bcl6 axis is critical to regulate osteoclast differentiation and bone homeostasis. J. Exp. Med. 207, 751-762. doi: 10.1084/ jem.20091957
Mizukami, J., Takaesu, G., Akatsuka, H., Sakurai, H., Ninomiya-Tsuji, J., Matsumoto, K., et al. (2002). Receptor activator of NF-kappaB ligand (RANKL) activates TAK1 mitogen-activated protein kinase kinase kinase through a signaling complex containing RANK, TAB2, and TRAF6. Mol. Cell Biol. 22, 992-1000. doi: 10.1128/MCB.22.4.992-1000.2002

Nishikawa, K., Nakashima, T., Hayashi, M., Fukunaga, T., Kato, S., Kodama, T., et al. (2010). Blimp1-mediated repression of negative regulators is required for osteoclast differentiation. Proc. Natl. Acad. Sci. U.S.A. 107, 3117-3122. doi: 10.1073/pnas.0912779107

Paiva-Fonseca, F., Santos-Silva, A. R., Della-Coletta, R., Vargas, P. A., and Lopes, M. A. (2014). Alendronate-associated osteonecrosis of the jaws: a review of the main topics. Med. Oral Patol. Oral Cir. Bucal 19, e106-e111. doi: 10.4317/ medoral.19094

Park-Min, K. H., Lee, E. Y., Moskowitz, N. K., Lim, E., Lee, S. K., Lorenzo, J. A., et al. (2013). Negative regulation of osteoclast precursor differentiation by $\mathrm{CD} 11 \mathrm{~b}$ and beta2 integrin-B-cell lymphoma 6 signaling. J. Bone Miner. Res. 28, 135-149. doi: 10.1002/jbmr.1739

Pihlstrom, B. L., Michalowicz, B. S., and Johnson, N. W. (2005). Periodontal diseases. Lancet 366, 1809-1820. doi: 10.1016/S0140-6736(05)67728-8

Sato, K., and Takayanagi, H. (2006). Osteoclasts, rheumatoid arthritis, and osteoimmunology. Curr. Opin. Rheumatol. 18, 419-426. doi: 10.1097/01.bor. 0000231912.24740.a5

Takayanagi, H., Kim, S., Koga, T., Nishina, H., Isshiki, M., Yoshida, H., et al. (2002). Induction and activation of the transcription factor NFATc1 (NFAT2) integrate RANKL signaling in terminal differentiation of osteoclasts. Dev. Cell 3, 889-901. doi: 10.1016/S1534-5807(02)00369-6

Teitelbaum, S. L. (2000). Bone resorption by osteoclasts. Science 289, 1504-1508. doi: $10.1126 /$ science.289.5484.1504

Teitelbaum, S. L. (2011). The osteoclast and its unique cytoskeleton. Ann. N. Y. Acad. Sci. 1240, 14-17. doi: 10.1111/j.1749-6632.2011.06283.x

Wada, N., Maeda, H., Yoshimine, Y., and Akamine, A. (2004). Lipopolysaccharide stimulates expression of osteoprotegerin and receptor activator of NF-kappa B ligand in periodontal ligament fibroblasts through the induction of interleukin1 beta and tumor necrosis factor-alpha. Bone 35, 629-635. doi: 10.1016/j.bone. 2004.04.023

Wong, B. R., Josien, R., Lee, S. Y., Vologodskaia, M., Steinman, R. M., and Choi, Y. (1998). The TRAF family of signal transducers mediates NF-kappaB activation by the TRANCE receptor. J. Biol. Chem. 273, 28355-28359. doi: 10.1074/jbc. 273.43.28355

Yagi, M., Miyamoto, T., Sawatani, Y., Iwamoto, K., Hosogane, N., Fujita, N., et al. (2005). DC-STAMP is essential for cell-cell fusion in osteoclasts and foreign body giant cells. J. Exp. Med. 202, 345-351. doi: 10.1084/jem.200 50645

Yoon, J. Y., Baek, C. W., Kim, H. J., Kim, E. J., Byeon, G. J., and Yoon, J. U. (2018). Remifentanil negatively regulates RANKL-induced osteoclast differentiation and bone resorption by inhibiting c-Fos/NFATc1 expression. Tissue Eng. Regen. Med. 15, 333-340. doi: 10.1007/s13770-018-0116-z

Zhao, B., and Ivashkiv, L. B. (2011). Negative regulation of osteoclastogenesis and bone resorption by cytokines and transcriptional repressors. Arthr. Res. Ther. 13:234. doi: 10.1186/ar3379

Zhao, B., Takami, M., Yamada, A., Wang, X., Koga, T., Hu, X., et al. (2009). Interferon regulatory factor- 8 regulates bone metabolism by suppressing osteoclastogenesis. Nat. Med. 15, 1066-1071. doi: 10.1038/ nm.2007

Conflict of Interest Statement: The authors declare that the research was conducted in the absence of any commercial or financial relationships that could be construed as a potential conflict of interest.

Copyright (c) 2019 Ihn, Lee, Lee, Bae, Kim, Jang, Bae, Shin and Park. This is an open-access article distributed under the terms of the Creative Commons Attribution License (CC BY). The use, distribution or reproduction in other forums is permitted, provided the original author(s) and the copyright owner(s) are credited and that the original publication in this journal is cited, in accordance with accepted academic practice. No use, distribution or reproduction is permitted which does not comply with these terms. 